\title{
The Be/X-ray binary LS 992/RX J0812.4-3114: Physical parameters and long-term variability
}

\author{
P. Reig ${ }^{1,2}$, I. Negueruela ${ }^{3}$, D. A. H. Buckley ${ }^{4}$, M. J. Coe ${ }^{5}$, J. Fabregat ${ }^{6}$, and N. J. Haigh ${ }^{5}$ \\ 1 Foundation for Research and Technology-Hellas, 71110 Heraklion, Crete, Greece \\ 2 Physics Department, University of Crete, 71003 Heraklion, Crete, Greece \\ 3 SAX SDC, Agenzia Spaziale Italiana, c/o Telespazio, via Corcolle 19, 00131 Roma, Italy \\ 4 South African Astronomical Observatory, PO Box 9, Observatory 7935, Cape Town, South Africa \\ 5 Physics \& Astronomy Department, Southampton University, SO17 1BJ, UK \\ 6 Astronomy \& Astrophysics Department. University of Valencia, 46100 Burjassot-Valencia, Spain
}

Received 2 August 2000 / Accepted 9 November 2000

\begin{abstract}
We present the first long-term optical and infrared study of the optical counterpart to the source RX J0812.4-3114, an X-ray pulsar with a Be type companion. During the period covered by the observations the profile of some Balmer lines changed from absorption to emission and back again to absorption. Contemporaneously, the infrared magnitudes varied by more than $0.8 \mathrm{mag}$. This long-term variability is interpreted as the formation and subsequent dissipation of the Be star's disc. The building up of the disc ended up in an active X-ray state characterised by regular outbursts occurring at 80 day intervals. The overall duration of the formation/dissipation of the disc is found to be $\lesssim 4.3$ years. Optical spectroscopic and infrared photometric observations were used to refine the spectral type of the primary (B0.2IV) and to monitor the circumstellar envelope around the Be star. $U B V R I$ and $u v b y \beta$ photometric observations allowed the determination of the astrophysical parameters of the optical companion.
\end{abstract}

Key words. stars: individual: LS 992/RX J0812.4-3114 - binaries: general - stars: pulsars: general stars: emission-line, Be - X-rays: stars

\section{Introduction}

The star LS 992 is the optical counterpart to the X-ray source RX J0812.4-3114. Such an association was originally proposed by Motch et al. (1997), as a result of a systematic cross-correlation between the ROSAT All Sky Survey (Voges et al. 1999) and several OB star catalogues in the SIMBAD database. The X-ray light curve of LS 992/RX J0812.4-3114 is characterised by 31.88 second pulsations, while the X-ray spectrum is best represented by an absorbed power-law component with a exponentially cut-off (Reig \& Roche 1999). In December 1997 the source made a transition from a quiescent state to a flaring state (Corbet \& Peele 2000), in which regular flares separated by 80 day intervals were detected with the All-Sky Monitor (ASM) onboard the Rossi X-ray Timing Explorer. Corbet \& Peele (2000) attributed the origin of these flares

Send offprint requests to: P. Reig,

e-mail: pablo@physics.uoc.gr

* Based on observations collected at the South African Astronomical Observatory, the European Southern Observatory, Chile (ESO N64.H-0059) and the Teide Observatory, Tenerife (Spain). to the periastron passage of the neutron star, hence this periodicity was naturally associated with the orbital period. The peak X-ray luminosity during outbursts was estimated to be of the order of $2.310^{36} \mathrm{erg} \mathrm{s}^{-1}$, assuming a distance to the source of $9 \mathrm{kpc}$.

In the optical band, LS 992 appears as a relatively bright $V \sim 12.4$ B0-1III-V star (Reed 1990). The optical spectra revealed $\mathrm{H} \alpha$ in emission and evidence for shortterm ( weeks) $V / R$ variability (Motch et al. 1997). The combination of an emission line B star plus a neutron star, whose existence is inferred from the characteristics of the $\mathrm{X}$-ray emission, defines a Be/X-ray binary. Be/X-ray binaries comprise approximately $70 \%$ of the more general class of high mass X-ray binaries, the remaining $\sim 30 \%$ contain evolved (luminosity class I and II) primaries. The high energy radiation is the result of accretion of the material expelled by the Be star onto the neutron star.

In this work we present the first long-term study of the optical counterpart of RX J0812.4-3114 and discuss the consequences of the correlated behaviour in the optical, infrared and X-ray wavelength bands. 
Table 1. Journal of the optical spectroscopic observations

\begin{tabular}{lcccc}
\hline Date & MJD & $\begin{array}{c}\text { Wavelength } \\
\text { coverage }(\AA)\end{array}$ & $\begin{array}{c}E W(\mathrm{H} \alpha) \\
(\AA)\end{array}$ & $\begin{array}{c}E W(\mathrm{H} \beta) \\
(\AA)\end{array}$ \\
\hline \multicolumn{5}{c}{ low-resolution spectra } \\
\hline $07-10-96$ & 50364 & $3200-8000$ & $-6.5 \pm 1.0$ & $-0.6 \pm 0.4$ \\
$05-02-98$ & 50850 & $3800-7800$ & $-16.8 \pm 0.8$ & $-1.4 \pm 0.5$ \\
\hline \multicolumn{5}{c}{ red-end spectra } \\
\hline $04-04-96$ & 50178 & $6300-7000$ & $+1.4 \pm 0.5$ \\
$03-02-98$ & 50848 & $6300-7000$ & $-17.0 \pm 1.0$ \\
$09-01-99$ & 51188 & $6200-6900$ & $-20.5 \pm 0.8$ \\
\hline \multicolumn{5}{c}{ blue-end spectra } \\
\hline $05-04-96$ & 50179 & $4200-5000$ & $+1.6 \pm 0.4$ \\
$05-03-98$ & 50878 & $3700-5200$ & $-1.4 \pm 0.3$ \\
$10-01-99$ & 51189 & $3700-5500$ & $-2.2 \pm 0.3$ \\
$31-10-99$ & 51483 & $3800-5000$ & $-1.8 \pm 0.1$ \\
$15-09-00$ & 51803 & $3000-5300$ & $+2.1 \pm 0.1$ \\
\hline
\end{tabular}

\section{Observations}

Blue spectroscopy of the source was obtained on 31st October 1999 using the $1.52-\mathrm{m}$ telescope at La Silla Observatory, Chile. The telescope was equipped with the Boller \& Chivens spectrograph + \#32 holographic grating and the Loral 38 camera. This configuration gives a resolution of $\sim 0.5 \AA /$ pixel. Measurements of arc line $F W H M$ indicate a spectral resolution of $\approx 1.4 \AA$ at $\sim 4500 \AA$. A second spectrum was obtained on 15 th September 2000, this time using holographic grating \#33, which gives a resolution of $\sim 1.0 \AA /$ pixel $(F W H M \approx 3.0 \AA$ at $\sim 4500 \AA)$.

All other spectra were taken using the $1.9 \mathrm{~m}$ telescope at SAAO, South Africa equipped with the Cassegrain spectrograph. The 1996 data were obtained using the Reticon Photon Counting System (RPCS), whereas all subsequent data were obtained using the SITe2 CCD detector. Details of the wavelength coverage achieved in each data set are given in Table 1.

The optical photometric data were acquired at the SAAO 1.0-m telescope using the Tek8 CCD camera, employing $U B V R_{\mathrm{C}} I_{\mathrm{C}}$, uvby $\beta$ and $\mathrm{H} \alpha$ filters, at a plate scale of 0.35 arcsec pixel ${ }^{-1}$ for the January 1999 observations and 0.7 arcsec pixel ${ }^{-1}$ for the October 1996 observations. The CCD frames were pre-processed using IRAF's CCDPROC package for bias subtraction, overscan removal and flat-fielding. Aperture photometry was performed in the STARLINK GAIA package, using observations of E-region standard stars (Menzies et al. 1989) for calibration to the Strömgren system.

The infrared photometric data were obtained at two telescopes - the $1.9 \mathrm{~m}$ equipped with the MkIII IR photometer at SAAO, in South Africa and the $1.5 \mathrm{~m}$ Telescopio Carlos Sánchez (TCS), Tenerife, Spain using the Continuously Variable Filter (CVF).

\section{Spectral type}

The spectra of LS 992 in the classification region are displayed in Fig. 1. The September 2000 spectrum shows no evidence for emission components, but it has a lower resolution and Signal-to-Noise Ratio (SNR) than the October 1999 spectrum. It has been included, however, so that the obvious changes can be observed. In particular, we call attention to the very strong in-filling of the He I lines at $\lambda \lambda 4026,4471,4921 \AA$ typical of Be/X-ray binary counterparts. In the October 1999 spectrum $\mathrm{H} \beta$ is strongly in emission, with an asymmetric blue-dominated profile, while similarly asymmetric emission components are present in the cores of all Balmer lines down to $\mathrm{H} \epsilon$. Longwards of $\lambda \sim 4750 \AA$ the continuum is dominated by weak (presumably Fe II) emission features.

Photospheric lines are all shallow and broad indicating a fast rotator. However, given the high SNR of the spectrum even the weakest metallic lines are detected. As is typical of Be/X-ray binary counterparts, the presence of weak He II lines indicates a spectral class close to B0. In order to improve the classification we have compared the spectrum of the source with those of standard stars taken either at the same resolution with the same instrument or at higher resolution (from the list of Steele et al. 1999).

We have used the main sequence standards $\nu$ Ori $(\mathrm{B} 0 \mathrm{~V}), \tau$ Sco $(\mathrm{B} 0.2 \mathrm{~V})$ and $o$ Per $(\mathrm{B} 0.5 \mathrm{~V})$. The absence of He II $\lambda 4200 \AA$ at this resolution indicates that the star is later than B0. If the star was in the main sequence, it would indicate a spectral type B0.5V or later. However, the strength of He II $\lambda 4686 \AA$ when compared to the nearby O II $\lambda 4676 \AA$ feature and the detectability of He II $\lambda 4541 \AA$ argue against this later classification. This seems to indicate a higher luminosity class.

In Fig. 1, the spectrum of LS 992 is shown together with those of the MK standard stars HD 48434 (B0III) and 1 Cas (B0.5III). The smooth progression of all the He II lines favours a spectral classification B0.2III for LS 992. However, some metallic lines seem weaker than expected and a luminosity class IV sounds more appropriate. Direct comparison with the spectrum of the B0.2IV standard $\phi^{1}$ Ori at a slightly lower resolution (from the digital atlas of Walborn \& Fitzpatrick 1990) supports this interpretation. Therefore we will accept a spectral type B0.2IV, though B0.2III cannot be excluded.

\section{Astrophysical parameters}

The determination of the astrophysical parameters by means of photometric calibrations in a Be star is not as straightforward as in a non-emission line B-type star due to the presence of the surrounding envelope, which distorts the characteristic photometric spectrum. Thus one has to correct for both circumstellar and interstellar reddening before any calibration can be applied. We have made use of the iterative procedure developed by Fabregat \& Torrejón (1998, hereafter FT98) to determine the intrinsic magnitudes. This method is based on the fact that there exists a correlation between the equivalent widths of the Balmer lines and the anomalies in the uvby photometric indices produced by continuum circumstellar emission. 
Table 2. Journal of photometric observations

\begin{tabular}{|c|c|c|c|c|c|c|c|c|c|c|c|c|}
\hline \multicolumn{13}{|c|}{ optical observations } \\
\hline Date & MJD & Telescope & $I$ & $I_{\text {err }}$ & $R$ & $R_{\text {err }}$ & $\bar{V}$ & $V_{\text {err }}$ & $\bar{B}$ & $\overline{B_{\text {err }}}$ & $\bar{U}$ & $U_{\text {err }}$ \\
\hline 05-10-1996 & 50362 & SAAO1.0 m & 11.72 & 0.02 & 12.16 & 0.02 & 12.48 & 0.02 & 12.86 & 0.02 & 12.57 & 0.09 \\
\hline Date & MJD & Telescope & $y$ & $y_{\text {err }}$ & $b$ & $b_{\text {err }}$ & $v$ & $v_{\text {err }}$ & $u$ & $u_{\text {err }}$ & $\beta$ & $\beta_{\text {err }}$ \\
\hline 23-01-1999 & 51202 & SAAO1.0 m & 12.52 & 0.04 & 12.89 & 0.04 & 13.11 & 0.07 & 13.33 & 0.14 & 2.47 & 0.02 \\
\hline \multicolumn{13}{|c|}{ infrared observations } \\
\hline Date & MJD & Telescope & $J$ & $J_{\text {err }}$ & $H$ & $H_{\text {err }}$ & $K$ & $\overline{K_{\mathrm{err}}}$ & $L^{\prime}$ & $L_{\text {err }}^{\prime}$ & & \\
\hline 13-01-1996 & 50096.56 & TCS & 11.39 & 0.05 & 11.14 & 0.02 & 10.84 & 0.02 & & & & \\
\hline 06-02-1998 & 50851.40 & SAAO1.9 m & 11.21 & 0.01 & 10.95 & 0.01 & 10.70 & 0.02 & 10.36 & 0.21 & & \\
\hline 07-02-1998 & 50852.51 & SAAO1.9 m & 11.25 & 0.01 & 11.01 & 0.01 & 10.66 & 0.07 & & & & \\
\hline 08-02-1998 & 50853.43 & SAAO1.9 m & 11.26 & 0.02 & 11.02 & 0.01 & 10.77 & 0.02 & 10.61 & 0.63 & & \\
\hline 28-10-1998 & 51115.76 & TCS & 11.67 & 0.03 & 11.31 & 0.02 & 11.03 & 0.04 & & & & \\
\hline 05-01-1999 & 51184.53 & $\mathrm{SAAO} 1.9 \mathrm{~m}$ & 11.54 & 0.02 & 11.25 & 0.07 & 10.95 & 0.02 & & & & \\
\hline 22-01-2000 & 51566.59 & TCS & 12.01 & 0.08 & 11.89 & 0.04 & 11.43 & 0.01 & & & & \\
\hline 22-01-2000 & 51566.593 & TCS & 12.04 & 0.33 & 11.78 & 0.14 & 11.25 & 0.02 & & & & \\
\hline
\end{tabular}

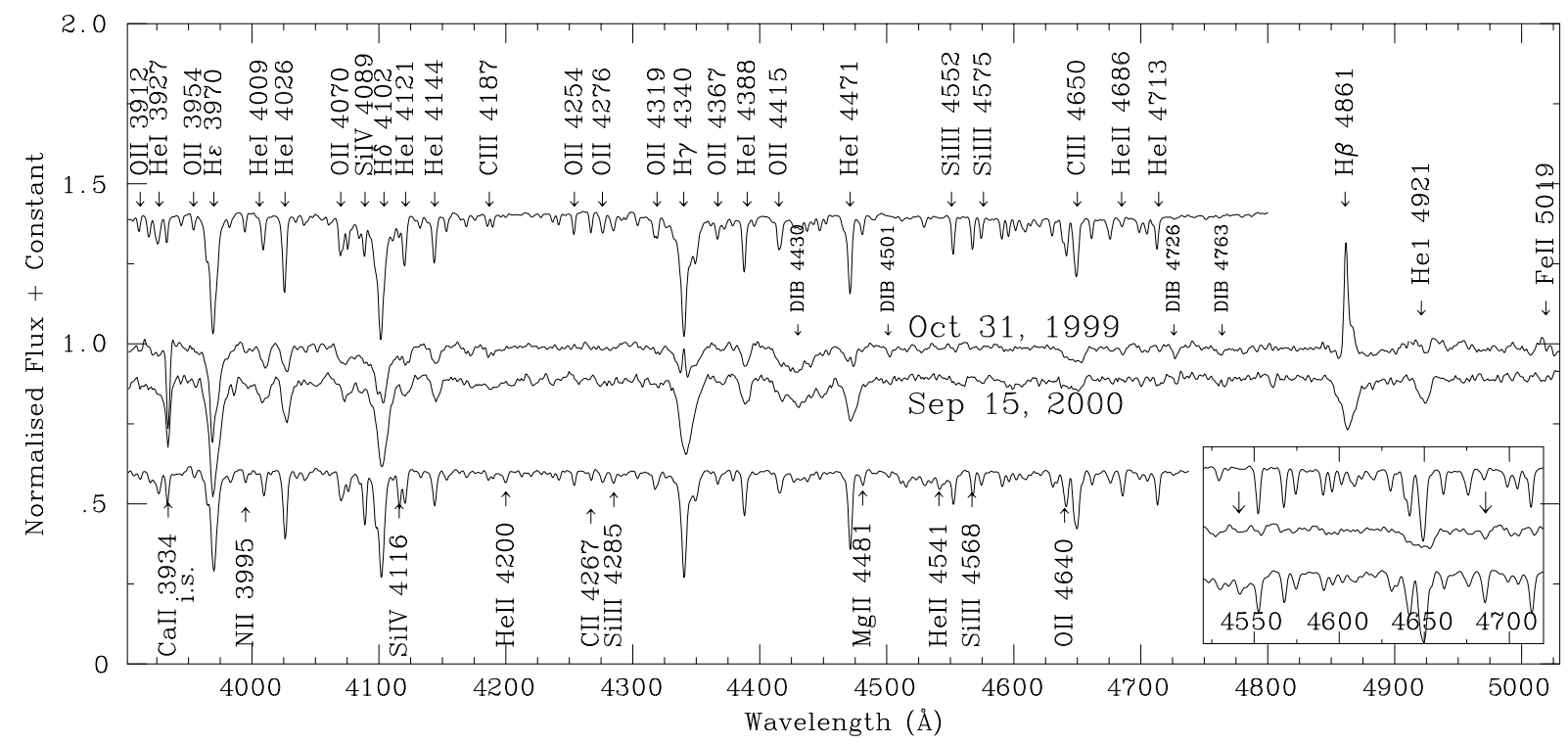

Fig. 1. The spectrum of LS 992 in the classification region (middle) is compared to those of the MK standard stars HD 48434 (B0III, bottom) and 1 Cas (B0.5III, top). The inset shows the smooth progression of HeII $\lambda \lambda 4541,4686 \AA$ (marked with arrows) when compared to the neighbouring Si III $\lambda 4553 \AA$ and O II $\lambda 4676 \AA$. All spectra have been smoothed with a $\sigma=0.8$ Gaussian filter, normalised by division into a spline fit to the continuum and offset for display

\subsection{Extinction, absolute magnitude and distance}

By applying the FT98 method we are able to disentangle the relative contributions of the interstellar and circumstellar extinction: $E^{\text {is }}(b-y)=0.433 \pm 0.033$ and $E^{\mathrm{cs}}(b-y)=0.051 \pm 0.025$, respectively. The errors reflect the accuracy of the FT98 procedure. Using the relation $E^{\text {is }}(B-V)=1.35 E^{\text {is }}(b-y)$ for interstellar extinction (Crawford \& Mandwewala 1976) and $E^{\mathrm{cs}}(B-V)=$ $1.20 E^{\mathrm{cs}}(b-y)$ for circumstellar extinction (Fabregat \& Reglero 1990) we find a total value for the extinction of $E(B-V)=0.65 \pm 0.05$, which is in good agreement with the values of 0.69 (from optical photometry) and $0.8 \pm 0.2$ (from interstellar spectral lines) reported by Motch et al. (1997). Correcting the $B-V$ index for extinction we find -0.27 , consistent with a B0.2IV star (Wegner 1994).
Although the $\beta$ index (Crawford \& Mander 1966) provides a measure of the luminosity for $\mathrm{O}$ and $\mathrm{B}$ stars it is strongly affected by emission. Thus it cannot be used to calibrate the stellar luminosity. In view of the good quality of our spectrum we prefer to assume the luminosity class derived from the spectral analysis and take $M_{V}=-4.3 \pm 0.5$ as would correspond for a B0.2 giantsubgiant star. The error has been arbitrarily taken so that it includes disagreements between different $M_{V}$ calibrations. The distance, estimated from the distance-modulus relation is found to be $d \approx 8.8 \mathrm{kpc}$.

\subsection{Effective temperature}

A B0.2IV star has a temperature close to $30000 \mathrm{~K}$ (Panagia 1973; Lamers 1981; Vacca et al. 1996). In O and 
Table 3. Astrophysical parameters of LS 992

\begin{tabular}{ll}
\hline Spectral type & B0.2IV-III \\
\hline$E(B-V)$ & $0.65 \pm 0.05$ \\
$T_{\text {eff }}$ & $28000 \pm 2000 \mathrm{~K}$ \\
Radius & $10 \pm 2 R_{\odot}$ \\
Mass & $17 \pm 3 M_{\odot}$ \\
$M_{V}$ & $-4.3 \pm 0.5$ \\
Distance & $8.8 \pm 4.0 \mathrm{kpc}$ \\
$B C$ & $-2.8 \pm 0.2$ \\
$M_{\text {bol }}$ & $-7.1 \pm 0.5$ \\
$\log g$ & $3.7 \pm 0.2 \mathrm{~cm}^{2} \mathrm{~s}^{-1}$ \\
$v \sin i$ & $240 \pm 20 \mathrm{~km} \mathrm{~s}^{-1}$ \\
\hline
\end{tabular}

B type stars the index $c_{0}$ is closely related to the effective temperature (Crawford 1978). Using the calibration $\log T_{\text {eff }}=0.186 c_{0}^{2}-0.580 c_{0}+4.402$ (Reig et al. 1997a) we obtain $T_{\text {eff }}=25400 \pm 4500 \mathrm{~K}$, where the error was obtained by propagating the photometric error. Due to the uncertainty in the $u$ measurement, hence $c_{0}$, the value of the temperature is not well constrained. Also, possible residual circumstellar emission may result in slightly larger $c_{0}$ values. If the intrinsic colour derived above, $(B-V)_{0}=-0.27$, is used in combination with the calibration of Gulati et al. (1989) a value of $26400 \pm 2300 \mathrm{~K}$ is found. Whereas if Eq. (11) of Napiwotzki et al. (1993) is applied, $T_{\text {eff }} \approx 29500 \mathrm{~K}$. As a compromise, we adopted a temperature of $28000 \pm 2000 \mathrm{~K}$.

Directly related to the effective temperature there is the bolometric correction, $B C=M_{\mathrm{bol}}-M_{V}$. The calibrations of $B C$ in terms of $T_{\text {eff }}$ result in $B C=-2.8 \pm 0.2$ (Balona 1994; Vacca et al. 1996).

\subsection{Radius}

In order to estimate the radius of LS 992 we have rewritten the luminosity equation $L=4 \pi \sigma R^{2} T_{\text {eff }}^{4}$ in terms of the visual brightness parameter $F_{V}$ (Barnes \& Evans 1976) and the $(b-y)_{0}$ colour index. With the value of the reddening derived in Sect. 4.1 we obtain $(b-y)_{0}=$ -0.115 which gives $F_{V}=4.166 \pm 0.026$ according to Eq. (6) of Moon (1984), where the error is that of the least-squares fit of the calibration. Substituting in $F_{V}=$ $4.236-0.1 M_{V}-0.5 \log \left(R / R_{\odot}\right)$ (Moon 1985) we finally obtain $R=10.0 \pm 2.6 R_{\odot}$. The final error was obtained by propagating the errors through the luminosity equation. This value compares well to the average radii in terms of MK spectral types calculated by Moon (1985), who give $12 R_{\odot}$ and $7.6 R_{\odot}$ for B0III and B1III stars, respectively. And also to those of Vacca et al. (1996) who derived 8 and $14.8 R_{\odot}$ for $\mathrm{B} 0.5 \mathrm{~V}$ and $\mathrm{B} 0.5 \mathrm{III}$ stars, respectively.

\subsection{Mass and gravity}

With the values of the temperature and bolometric luminosity, we can estimate the mass from evolutionary models. We have used the interpolation formula given by Balona (1994) for the Claret \& Giménez (1992) models

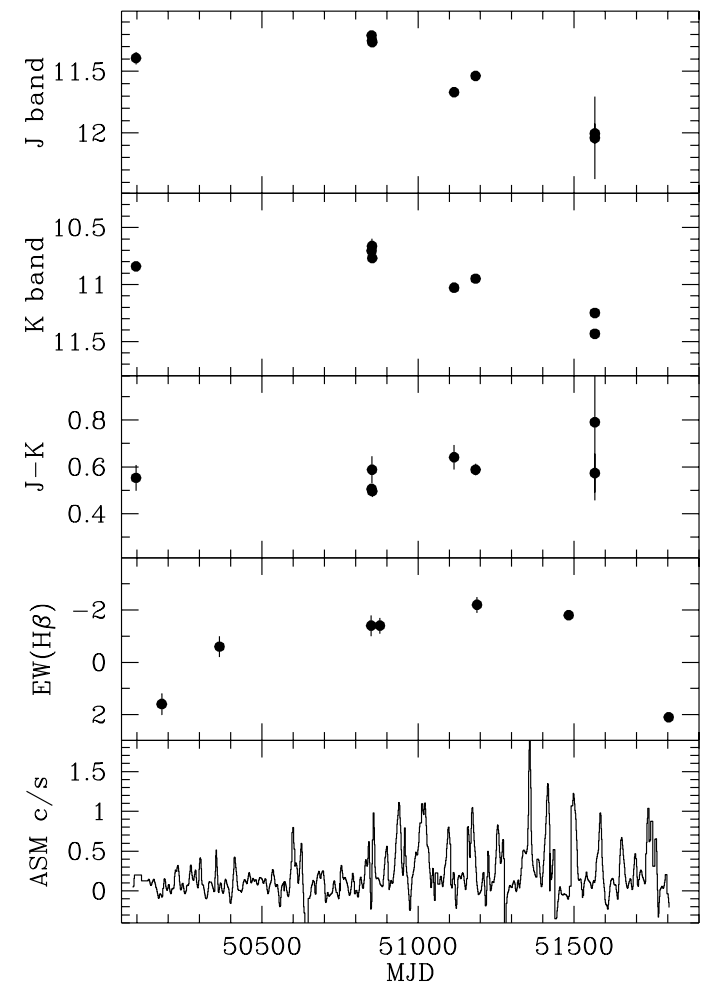

Fig. 2. IR magnitudes, $\mathrm{H} \beta$ equivalent width and ASM light curve for the period April 1996-September 2000. The activity of the source increased suddenly at around 1997 December (MJD 50800)

and found $M=17 \pm 3 M_{\odot}$. Once we know the mass and radius of the star we can calculate its gravity by means of $\log g=4.44+\log M-2 \log R$, where $M$ and $R$ must be given in solar units. The result is $\log g=3.7 \pm 0.2 \mathrm{~cm} \mathrm{~s}^{-1}$.

\subsection{Rotational velocity}

The projected rotational velocity $v \sin i$ can be estimated from the width of the spectral lines. However, in practice, it is difficult to find clean spectral lines, i.e., not affected by circumstellar emission. The spectrum taken in September 2000 shows Balmer lines in absorption, indicating a discloss phase. In addition, He I lines are mainly formed in the star's photosphere and are expected to have the smallest disc contribution. We have used the He I $\lambda 4026$, He I $\lambda 4144$ and He I $\lambda 4388$ lines and obtained $F W H M=6.1,5.9$ and $7.0 \AA$, respectively.

From Buscombe's (1969) approximation

$\frac{v \sin i}{c}=\frac{F W H M}{2 \lambda_{0}(\ln 2)^{1 / 2}}$

where $\lambda_{0}$ is the rest wavelength of the line and $F W H M$ represents the full width at half maximum corrected for instrumental broadening, we obtain $v \sin i=240 \mathrm{~km} \mathrm{~s}^{-1}$, $220 \mathrm{~km} \mathrm{~s}^{-1}$ and $260 \mathrm{~km} \mathrm{~s}^{-1}$ for He I $\lambda 4026, \lambda 4144$ and $\lambda 4388$ respectively. Similar values are found using the fits of Steele et al. (1999). 


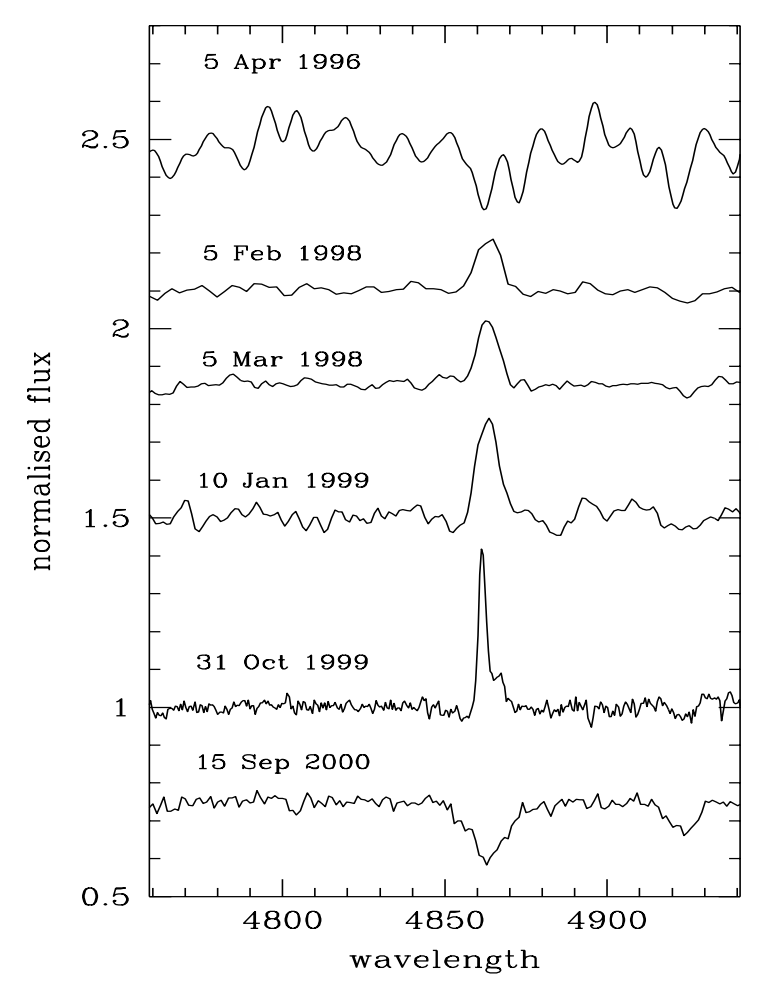

Fig. 3. The large line profile variability indicates major structural changes in the disc of the Be star. The continuum has been normalised to unity and the spectra offset for display

\section{Long-term variability}

Prior to 1998, LS 992/RX J0812.4-3114 was in an Xray dormant state as indicated by the fact that the flux from the All Sky Monitor (ASM), onboard the Rossi X-ray Timing Explorer (RXTE), was consistent with the X-ray background flux, $\sim 0.1 \mathrm{c} / \mathrm{s}$ (Fig 2). At the end of 1997 (MJD 50800) the source underwent a transition from this inactive state to a flaring state, consisting of regular outbursts occurring at approximately 80 day intervals (Corbet \& Peele 2000). At the onset of the X-ray activity the optical companion was in a bright infrared and optical state.

In Be/X-ray binary systems the optical primary has a circumstellar disc, which is presumably made up of material expelled by the $\mathrm{B}$ star. A neutron star orbits around the Be star in a moderately eccentric orbit. The ultimate physical process which leads to mass ejection in Be stars is unknown but it is probably one (or a combination) of these: radiation pressure, centrifugal force, thermal expansion and hydromagnetic waves (Strafella et al. 1998; Balona 2000). This circumstellar disc is responsible for the two defining observational properties of a Be star, namely the infrared excess and the emission lines. The mechanism that powers the X-ray source is accretion onto a neutron star and the fuel is the material in the disc.

In this scenario we would expect some type of correlation between the X-ray and the optical/infrared emission. Figure 2 shows the ASM and infrared light curves together with the evolution of the $\mathrm{H} \beta$ equivalent width. When the circumstellar disc becomes large enough the neutron star is able to interact with it and produce X-rays. As can be seen in Fig. 2 the equivalent width of the $\mathrm{H} \beta$ line, $E W(\mathrm{H} \beta)$ increased slowly during the years prior to the onset of the X-ray activity, indicating the building-up of the disc. Spectral hydrogen lines were in absorption in April 1996, in emission in October 1996 and back to absorption in September 2000. The absorption profile indicates that the Be star's disc was at some point non existent. Subsequently, the disc began to grow until it was large enough for the neutron star to interact. The source was X-ray active in December 1997. Thus, the build-up of the disc took $\lesssim 18$ months. The source reached an optical and infrared maximum and then began to decrease.

The issue of how far/big the disc can grow was discussed by Reig et al. (1997b), who concluded that the disc may be truncated by the continuous passage of the neutron star once the disc radius is comparable to the periastron distance. This effect is expected to be stronger in systems with short orbital periods. Assuming that the 80 day periodicity found in the X-rays (Corbet \& Peele 2000) represents the orbital period, the diagram $E W(\mathrm{H} \alpha)-P_{\text {orb }}$ (Reig et al. 1997b) predicts $E W_{\max }$ between -20 to $-25 \AA$, i.e, close to the maximum value reported in our long-term monitoring of the system (Table 1). Thus, it seems reasonable to think that the disc in LS 992/RX J0812.4-3114 reached its maximum size by the time that the X-ray emission began.

The onset of the X-ray activity marked the beginning of the disc-loss phase. This result can be understood since the material from the disc constitutes the fuel that powers the X-ray source. The disc is also responsible for the infrared excess observed in Be stars, which is attributed to recombination radiation from ionized hydrogen (freefree and free-bound processes). Any major change in the properties of the disc will translate into the infrared magnitudes. Soon after the onset of the X-ray emission, the infrared magnitudes began to decrease and by the time $\mathrm{H} \beta$ line went back into absorption $J, H$ and $K$ had decreased by more than 0.8 magnitudes. This relationship between $\mathrm{X}$-ray and infrared emission also demonstrates that the compact object exerts a notable influence on the fate of the Be star's disc.

Overall, the entire episode of formation and dissipation of the disc, considering it as the time elapsed between two consecutive absorption line phases, took $\lesssim 4.3$ years (Fig. 3). It is interesting to note that the $\mathrm{H} \alpha$ line was showing little emission $(E W(\alpha)=-4.6 \AA)$ back in April 18, 1992 (Motch et al. 1997), that is $\sim 4$ years before the first measurement reported here. That state must correspond with the first stages of a previous active state, and implies that the $4.3 \mathrm{yr}$ period is repeatable. In other words, the formation and loss of the disc represents a cyclic event in LS 992/RX J0812.4-3114.

The X-ray periodic flaring behaviour is common among Be/X-ray binaries and the flares, which normally peak at around periastron, are referred to as type I outbursts. They differentiate from type II outbursts which are major increases $\left(10^{2}-10^{3}\right)$ in X-ray flux and tend to peak 
at later orbital phase. Many of the giant outbursts are detected in the middle or at the beginning of a sequence of normal outbursts (Bildsten et al. 1997). The case of LS 992/RX J0812.4-3114 is different since a series of normal outbursts followed immediately the inactive phase. In this respect LS 992/RX J0812.4-3114 resembles EXO 2030 +375 . This $46 \mathrm{~d}$ orbital period Be/X-ray binary was in an active X-ray state, between April 1991 (when BATSE became operative) until August 1993. It switched off for three years to reappear in April 1996, showing periodic X-ray outbursts at $46 \mathrm{~d}$ intervals (Reig \& Coe 1998a,b). The similar X-ray luminosities of the flares $\left(2-410^{36} \mathrm{erg} \mathrm{s}^{-1}\right)$ stresses the resemblance of these two systems.

While the correlation between optical and infrared emission is the expected behaviour for a growing disc the long-term monitoring of Be/X-ray binaries is providing new insights into the way the neutron star affects the properties of the disc and the generation of high energy radiation. The traditional idea of expanding discs is being replaced by disc dissipation/reformation cycles which control the X-ray behaviour. Once the disc reaches a certain size, which presumably is limited by the continuous passages of the neutron star, the disc becomes more dense and unstable and material is fed onto the neutron star. Negueruela et al. (2000) have pointed out that disc truncation by the neutron star may indirectly cause the warping/tilting of the Be star's disc. They applied the viscous decretion disc model (Negueruela \& Okazaki 2000) to the Be/X-ray transient 4U 0115+63/V635 Cas to explain its $\mathrm{X}$-ray and optical/IR behaviour. 4U 0115+63/V635 Cas presented a sizable disc $\sim 6$ months after being observed discless and was X-ray active only 13 months after a photometric low. These timescales compare very well with those in LS 992/RX J0812.4-3114.

The asymmetry of the $\mathrm{H} \beta$ line profiles in LS 992 and the variability of its blue $(V)$ and red $(R)$ peaks (Fig. 3) are reminiscent of the so-called $V / R$ variability, and point toward a perturbed density distribution in the disc. The perturbation is associated with the existence of global oscillation modes propagating though the disc (Okazaki 1991, 1997; Papaloizou et al. 1992). The $V / R$ pattern developed shortly after the disc build-up began, supporting the idea that the discs are intrinsically unstable against density perturbations.

\section{Conclusion}

We have witnessed the appearance and disappearance of the circumstellar envelope in the $\mathrm{Be} / \mathrm{X}$ ray binary LS 992/RX J0812.4-3114. Using UBVRI and uvby $\beta$ photometric observations we have derived the astrophysical parameters of the Be/X-ray binary LS 992/RX J0812.43114. The optical companion turns out to be a B0.2IVe star showing marked optical and infrared variability on timescales of months to years. The reversal from positive to negative and then back to positive of the equivalent width of the $\mathrm{H} \beta$ line, the large amplitude of variation of both, the $\mathrm{H} \alpha$ equivalent width $(\Delta E W(\mathrm{H} \alpha) \approx 20 \AA)$ and the infrared magnitudes $(\Delta J \approx \Delta H \approx \Delta K \approx 0.8 \mathrm{mag})$ are interpreted as the formation and dissipation of the Be star's disc. The long-term monitoring of LS 992/RX J0812.4-3114 constrains the timescale for formation and dissipation of the disc to about 4.3 years. Measurements found in the literature seem to indicate that this episode is cyclic and basically supports the picture of dynamically unstable discs, that is, the discs go through cycles of dissipation/reformation which control the X-ray behaviour of the source.

Acknowledgements. P. Reig acknowledges partial support via the European Union Training and Mobility of Researchers Network Grant ERBFMRX/CT98/0195. IN was supported by an ESA external fellowship. This work made use of data provided by the RXTE ASM team at MIT and GSFC. We thank the referee, Dr. Berghoefer, for his useful comments.

\section{References}

Balona, L. 1994, MNRAS, 268, 11

Balona, L. 2000, in ASP Conf. Ser., The Be Phenomenon in Early-Type stars, ed. M. A. Smith, H. F. Henrichs, \& J. Fabregat

Barnes, T. G., \& Evans, D. S. 1976, MNRAS, 174, 489

Bildsten, L., Chakrabarty, D., Chiu, J., et al. 1997, ApJS, 113, 367

Buscombe, W. 1969, MNRAS, 144, 1

Claret, A. \& Giménez, A. 1992, A\&AS, 96, 255

Corbet, R. H. D., \& Peele, A. G. 2000, ApJ, 530, L33

Crawford, D. L. 1978, AJ, 83, 48

Crawford, D. L., \& Mander, J. 1966, AJ, 71, 114

Crawford, D. L., \& Mandwewala, N. 1976, PASP, 88, 917

Fabregat, J., \& Reglero, V. 1990, MNRAS, 247, 407

Fabregat, J., \& Torrejon, J. M. 1998, A\&A, 332, 643

Gulati, R. K., Malagnini, M. L., \& Morossi, C. 1989, A\&AS, 80,73

Lamers, H. J. G. L. M. 1981, ApJ, 245, 593

Menzies, J. W., Cousins, A. W. J., Banfield, R. M., \& Laing, J. D. 1989, SAAO Circ., 13, 1

Moon, T. 1984, MNRAS, 211, 21

Moon, T. 1985, Ap\&SS, 177, 261

Motch, C., Haberl, F., Dennerl, K., Pakull, M., \& JanotPacheco, E. 1997, A\&A, 323, 853

Napiwotzki, R., Schönberner, D., \& Wenske, V. 1993, A\&A, 268,653

Negueruela, I., \& Okazaki, A. T. 2000, A\&A, in press

Negueruela, I., Okazaki, A. T., Fabregat, J., et al. 2000, A\&A, in press

Okazaki, A. T. 1991, PASJ, 43, 75

Okazaki, A. T., 1997, A\&A, 318, 548

Panagia, N. 1973, AJ, 78, 929

Papaloizou, J. C., Savonije, G. J., \& Henrichs, H. F. 1992, A\&A, 265, L45 
Reed, C. B. 1990, AJ, 100, 737

Reig, P., \& Coe, M. J. 1998a, MNRAS, 294, 118

Reig, P., \& Coe, M. J. 1998b, MNRAS, 301, 42

Reig, P., \& Roche, P. 1999, MNRAS, 306, 95

Reig, P., Fabregat, J., Coe, M. J., et al. 1997a, A\&A, 322, 183

Reig, P., Fabregat, J., \& Coe, M. J. 1997b, A\&A, 322, 193

Steele, I. A., Negueruela, I., \& Clark, J. S. 1999, A\&AS, 137, 147
Strafella, F., Pezzuto, S., Corciulo, G. G., Bianchini, A., \& Vittone, A. A. 1998, ApJ, 505, 299

Voges, W., Aschenbach, B., Boller, T., et al. 1999, A\&A, 349, 389

Vacca, W. D., Garmany, C. D., \& Shull, J. M. 1996, ApJ, 460, 914

Walborn, N., \& Fitzpatrick, E. 1990, PASP, 102, 379

Wegner, W. 1994, MNRAS, 270, 229 